\title{
Qualitätskontrolle in der Hämatologie
}

W. Hohenwallner

Zentrallaboratorium des KH der Barmherzigen Schwestern, Linz

\begin{abstract}
Zusammenfassung:
In einem Übersichtsreferat werden die Möglichkeiten der internen und externen Qualitätskontrolle behandelt. Dabei wird neben der Verwendung von stabilisierten Kontrollbluten zur Überprüfung von Richtigkeit und Präzision für die interne hämatologische Qualitätskontrolle auf die Möglichkeiten der Erstellung des „daily mean” bzw. der Wiederholbarkeit von Patientenproben hingewiesen. In der externen Qualitätskontrolle werden regionale und nationale Rundversuche für die entsprechenden hämatologischen Parameter und deren Ergebnisse diskutiert.
\end{abstract}

Schlüsse/wörter:

Qualitätskontrolle interne und externe - Hämatologie - Ringversuche

Suminary:

A review shows possibilities of internal and external quality control systems. For internal quality control are indicated stabilized control blood specimen for testing precision and accuracy, besides the calculation of the daily mean and the repetition of patients specimen are recommended. For external quality control interlaboratory trials of the hematological parameters are discussed.

Keywords:

Quality control internal and external - hematology - interlaboratory trials

\section{Einleitung}

Ein Kontrollsystem für die Qualität der Untersuchungen in der klinischen Chemië, der Gerinnung und Hämatologie ist heute unumstritten. Mit dem technischen Fortschritt in der Messung von hämatologischen Parametern hat sich auch das Qualitätskontrollsystem entwickelt.

Im folgenden Übersichtsreferat sollen die verschiedenen Möglichkeiten zur Überprüfung der Richtigkeit und Präzision in der Hämatologie aufgezeigt werden.

\section{Interne Qualitätskōñ̈trolle}

Das dem Patienten am nächsten stehende Kontrollmaterial ist das Frischblut. Es erlaubt zwar Momentuntersuchungen im Vergleich mit der entsprechenden Referenzmethode und sollte als Grundlage für die Kalibration der automatischen Geräte gelten, die keine Absolutmessung durchführen können. Es eignet sich aber naturgemäß nicht für Untersuchungen über einen längeren Zeitraum.

Primäre Standards sind nur für die Messung des Hämoglobins erhältlich, für die Analyse der Teilchenmessung sind sekundäre Standards im Sinne von stabilisierten Kontrollbluten verwendbar.

Die zunächst eher begrenzte Haltbarkeit dieser Präparate auf ca. 30 Tage (z. B. CH-35, DADE) wurde verbessert und das Material weist heute eine hohe Stabilität von mindestens 2 Monaten auf (z.B. CH-60 DADE, 4-C-Coulter, Controll Blood Hyland, Asid etc.). Für die Erythrozytenbestimmung sind mit den stabilisierten Kontrollbluten kaum Schwierigkeiten verbunden, außer bei der Bestimmung des Hämatokrits mittels Leitfähigkeitsmessung. Hingegen muß man sich für die Leukozytenzählung mit stabilisierten Vogel-(Truthahn)erythrozyten auch heute noch zufriedengeben.

Die neuesten Präparate enthalten zusätzlich auch haltbare Thrombozyten. Nach Öffnen des Fläschchens verringert sich jedoch die Stabilität dieser Präparate auf 3 bis 7 Tage je nach Herstellerangaben.

Eigene Erfahrungen über Präzision und Richtigkeit bei der Messung der Erythrozyten, Leukozyten und des Hämoglobins an Hand des Kontrollblutes $\mathrm{CH}-35$ über einen Zeitraum von einem Jahr wurden 1975 publiziert (1).

Mittels halbautomatischer Messung am Coulter FN wurden im normalen und abnormalen Bereich für die Erythrozytenzählung VKs zwischen 1 und $2 \%$ von Tag zu Tag erzielt, für die Leukozytenzählung 3-7\% und für die Messung des Hämoglobins 1-3\%. Die Richtigkeit war in jedem Falle gegeben. Geprüft wurden insgesamt 21 Chargen dieses Präparates. Da das Kontrollblut wie Patientenblut behandelt wurde, konnte auch die präanalytische Phasé überwacht werden.

Mit Verlängerung der Stabilisierung des Kontrollmaterials auf 2 Monate wurde ein dritter Meßbereich mit abnormal tiefen Werten zusätzlich angeboten.

Wir untersuchten am Coulter S-jr. drei verschiedene Kontrollblute mit drei Meßbereichen (Control Blood-Dade, 4-CCoulter, Blood Control Hyland) und prüften Erythrozyten, Leukozyten, Hämoglobin und Hämatokrit. Es wurden von Tag zu Tag für die Erythrozyten VKs zwischen 1 und $3 \%$ für alle Präparate gemessen, für die Leukozyten $5-10 \%$, für das Hämoglobin 1,3-3,8\% und für den Hämatokrit 1,4-3,8\%.

Ein weiterer Fortschrjtt war die Entwicklung von Kontrollmaterial für die Thrombozytenzählung. An Hand von plätt- 
I Ievel 11
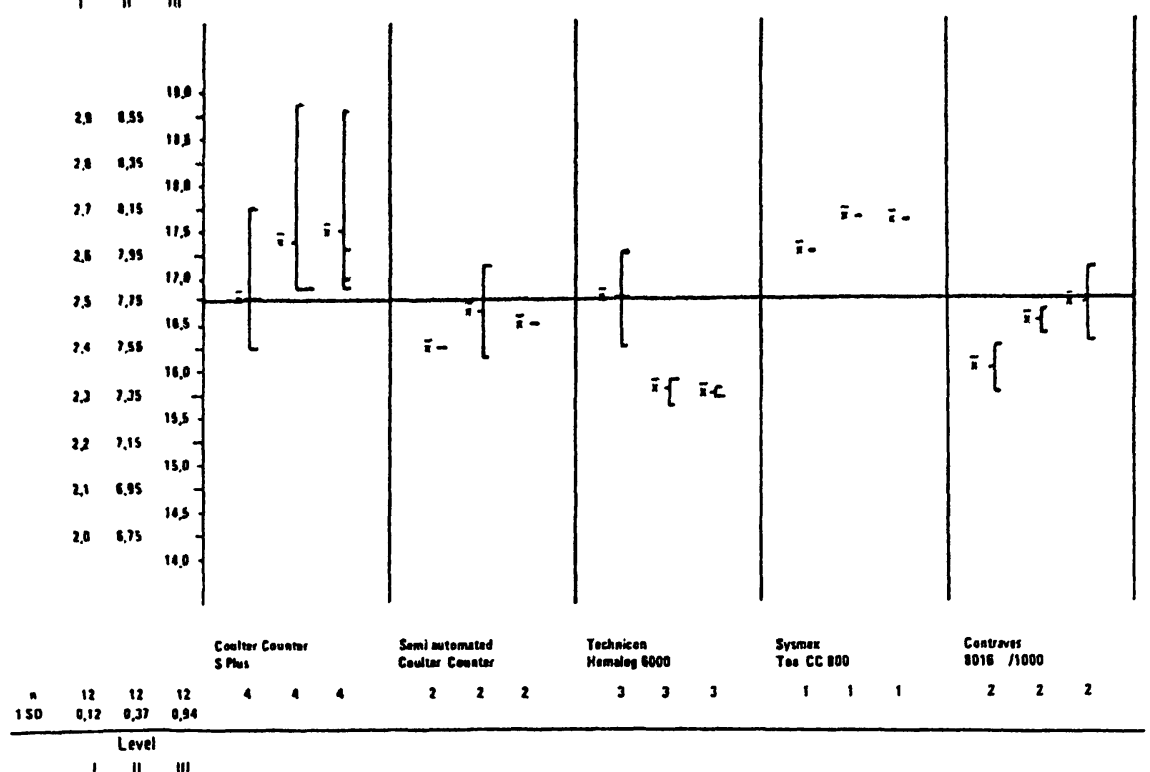

I II III

$2.75 \quad 5.15 \quad 1,2$

$\begin{array}{rrr}2.00 & 5.05 & 7.0 \\ 2,05 & 4.95 & 6.0\end{array}-$

$\begin{array}{lll}2,60 & 4,85 & 6,6\end{array}$

$2,55 \quad 4,75 \quad .6,4$

$\begin{array}{lll}2.50 & 0.65 & 62\end{array}$

$2.45 \quad 4.55$

$2.40 \quad 4.45 \quad 5.8$

$2,35 \quad 4,35 \quad 5,6]=$

$2.30 \quad 4.25 \quad 5.4$

$\begin{array}{lll}2.25 & 4.15 \quad 5,2\end{array}$

$2.20 \quad 4.05 \quad 5.0$

$2,15 \quad 3,95 \quad 40$

1

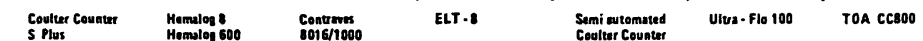

$\begin{array}{llllll}n & 23 & 23 & 23 & 4 & 4\end{array}$

9

22

Level

255 .

$10 \quad 255 \quad 455$

$70 \quad 235 \quad 415$

65225 395-

60215 375-

$55 \quad 205 \quad 35$

So 195 335

45 its 3is

$40 \quad 175 \quad 295$

$35 \quad 165 \quad 275$

$30 \quad 155 \quad 253$

$25 \quad 145 \quad 235$

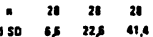

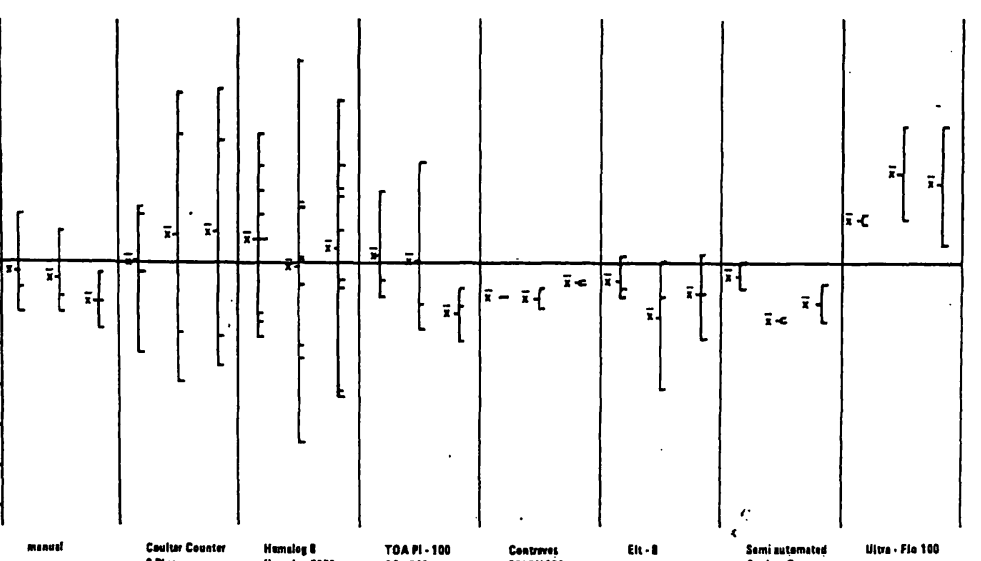

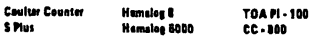

Abb. 1 : Ergebnisse des Feldversuchs der Firma Merz + Dade für Erythrozyten. gemessen an Hand des Control Blood Plus Lot No. 8982
Abb. 2: Ergebnisse des Feldversuchs der Firma Merz + Dade für Leukozyten gemessen an Hand des Control Blood Plus Lot No. 8982
Abb. 3: Ergebnisse des Feldversuchs der Firma Merz + Dade für

- Thrombozyten, gemessen an Hand des Control Blood Plus Lot No. 8982 
chenangereichertem menschlichen Plasma konnte auch eine Qualitätskontrolle für die Thrombozytenzählung bewerkstelligt werden. Wir untersuchten die Thrombozyten in der Kontrollsubstanz HAEM-PC (Firma Baker) normal und low am Counter Al 134 (Analysis Instruments), die diesbezüglichen Resultate wurden 1980 veröffentlicht (2). Wir fanden für Richtigkeit und Präzision ein zufriedenstellendes Ergebnis. Im Normalbereich von Tag zu Tag einen VK von $1,8-5,4 \%$, im abnormal tiefen Bereich 6,2-11,2\%.

Die nächste Entwicklungsstufe war ein Kontrollblut nicht nur für die Erythrozyten und Leukozyten, sondern auch für stabile Thrombozyten in einem Präparat.

Diese Entwicklung war notwendig, da auch der apparative Fortschritt aus einer Patientenprobe sämtliche hämatologischen Parameter in einem Arbeitsschritt bzw. einem Gerät zu verarbeiten, bereits Wirklichkeit wurde.

Derzeit sind eine Reihe solcher Produkte im Handel erhältlich, die mehr als 8 Parameter mit Sollwertangaben versehen, mit drei Meßbereichen beinhalten (z. B. 4-C-Coulter, Controll Blood Plus DADE und Hyland). Die Haltbarkeit ist bei nicht geöffneten Fläschchen auf ca. 2 Monate beschränkt. Nach Öffnen der Kontrollprobe werden unterschiedliche Angaben zwischen 3 und 7 Tagen vorgeschrieben.

Erste Informationen über das Kontrollblut „Control Blood Plus" mit 3 "levels” an Hand eines Apparatevergleichs wurden uns in dankenswerter Weise von der Fa. DADE überlassen. Diese Ergebnisse sind aus den Abb. 1-3 ersichtlich.

Bei diesen drei Graphiken wurde der Mittelwert berechnet aus den Resultaten aller Labors und der Mittelwert des einzelnen
Labors eingetragen. Um einen besseren Überblick zu bekommen, wurden die verschiedenen Geräte getrennt aufgeführt. Die Resultate für Ery-, Leuko- und Thrombozyten, bezogen auf den Richtwert, werden hauptsächlich durch folgende Faktoren beeinflußt: Linearität des Gerätes, Koinzidenzkorrektur und Kalibration.

\section{Tagesmittel (daily mean)}

Eine weitere Unterstützung der internen Qualitätskontrolle ist die Bestimmung des Tagesmittel für die hämatologischen Parameter. Wir erhalten in unserem Laboratorium über die EDV das über alle Daten und Methoden ermittelte Tagesmittel ohne Berücksichtigung der Extremwerte.

In der Tab. 1 sind über 5 Tage das Tagesmittel für Erythrozyten, Leukozyten, Hämoglobin und $\mathrm{HB}_{\mathrm{E}}$ und Thrombozyten zusammengefaßt. Nach Korrektur der Extremwerte wird bezogen auf die hämatologischen Referenzbereiche (3) das arithmetische Mittel des Sollbereiches der Referenz sehr gut getroffen.

Wiederholbarkeit von Patientenproben:

Die Wiederholbarkeitsprüfung von Patientenproben ist ein weiter Weg, die Güte des Materials (EDTA-blut) und das eingesetzte Zählgerät zu testen.

Wir untersuchten am Coulter S-jr. einige Patientenproben und wiederholten nach Lagerung des EDTA-Blutes im Kühlschrank bei $+4^{\circ} \mathrm{C}$ am darauf folgenden Tag die Messungen mit sehr gutem Ergebnis (siehe Tab. 2). Größere Schwankungen wurden bei den Thrombozytenzählungen auf unserem Gerät (Al Instrument) nachgewiesen.

Tab. 1: „daily mean” von Erythrozyten, Leukozyten, Thrombozyten, Hämoglobin und HB ${ }_{E}$ gemessen am Coulter S-jr. an 5 aufeinanderfolgenden Tagen ohne Korrektur der Extremwerte

\begin{tabular}{|c|c|c|c|c|c|}
\hline Tag & Erythrozyten & Hämoglobin & $H B_{E}$ & Leukozyten & Thrombozyten \\
\hline $\begin{array}{l}1 . \\
2 . \\
3 . \\
4 . \\
5 .\end{array}$ & $\begin{array}{l}4,61 \\
4,52 \\
4,49 \\
4,58 \\
4,46\end{array}$ & $\begin{array}{l}13,76 \\
13,45 \\
13,61 \\
13,83 \\
13,55\end{array}$ & $\begin{array}{l}29,95 \\
29,82 \\
30,45 \\
30,30 \\
29,99\end{array}$ & $\begin{array}{l}9,03 \\
8,14 \\
8,37 \\
8,03 \\
9,06\end{array}$ & $\begin{array}{l}236,38 \\
219,12 \\
214,80 \\
222,02 \\
209,03\end{array}$ \\
\hline
\end{tabular}

Tab. 2: Patienten-Proben (Wiederholbarkeit von Tag zu Tag) EDTA-Blut

\begin{tabular}{|c|c|c|c|c|c|c|}
\hline \multirow[t]{2}{*}{ Probe } & & \multicolumn{4}{|l|}{ Coulter-S jr. } & \multirow{2}{*}{$\begin{array}{l}\text { AJ-Instrument } \\
\text { Thrombozyten }\end{array}$} \\
\hline & & Erythrozyten & Hämoglobin & $H B_{E}$ & Leukozyten & \\
\hline 1. & $\begin{array}{l}\text { 1. Wert } \\
\text { 2. Wert }\end{array}$ & $\begin{array}{l}4,60 \\
4,60\end{array}$ & $\begin{array}{l}14,9 \\
15,0\end{array}$ & $\begin{array}{l}32,4 \\
32,6\end{array}$ & $\begin{array}{l}6,9 \\
7,4\end{array}$ & $\begin{array}{l}309 \\
320\end{array}$ \\
\hline 2. & $\begin{array}{l}\text { 1. Wert } \\
2 . \text { Wert }\end{array}$ & $\begin{array}{l}4,71 \\
4,72\end{array}$ & $\begin{array}{l}13,9 \\
13,9\end{array}$ & $\begin{array}{l}29,5 \\
29,2\end{array}$ & $\begin{array}{l}6,0 \\
6,2\end{array}$ & $\begin{array}{l}502 \\
318\end{array}$ \\
\hline 3. & $\begin{array}{l}\text { 1. Wert } \\
\text { 2. Wert }\end{array}$ & $\begin{array}{l}5,00 \\
4,96\end{array}$ & $\begin{array}{l}16,9 \\
16,8\end{array}$ & $\begin{array}{l}33,8 \\
33,8\end{array}$ & $\begin{array}{l}5,7 \\
5,4\end{array}$ & $\begin{array}{l}195 \\
199\end{array}$ \\
\hline 4. & $\begin{array}{l}\text { 1. Went } \\
\text { 2. Wert }\end{array}$ & $\begin{array}{l}4,75 \\
4,66\end{array}$ & $\begin{array}{l}14,4 \\
14,4\end{array}$ & $\begin{array}{l}30,3 \\
30,4\end{array}$ & $\begin{array}{l}20,4 \\
22,3\end{array}$ & $\begin{array}{l}275 \\
309\end{array}$ \\
\hline 5. & $\begin{array}{l}\text { 1. Wert } \\
\text { 2. Wert }\end{array}$ & $\begin{array}{l}5,52 \\
5,52\end{array}$ & $\begin{array}{l}13,5 \\
13,5\end{array}$ & $\begin{array}{l}24,4 \\
24,4\end{array}$ & $\begin{array}{l}6,2 \\
7,4\end{array}$ & $\begin{array}{l}296 \\
320\end{array}$ \\
\hline 6. & $\begin{array}{l}\text { 1. Wert } \\
\text { 2. Wert }\end{array}$ & $\begin{array}{l}4,45 \\
4,50\end{array}$ & $\begin{array}{l}13,6 \\
13,6\end{array}$ & $\begin{array}{l}30,5 \\
30,1\end{array}$ & $\begin{array}{l}5,7 \\
5,6\end{array}$ & $\begin{array}{l}305 \\
355\end{array}$ \\
\hline
\end{tabular}




\section{Externe Qualitätskontrolle}

Wir möchten diesen Abschnitt in die Problematik der regionalen und nationalen Rundversuche teilen.

Regionale Rundversuche:

Im Jahre 1974 wurde ein Rundversuch auf regionaler Ebene über 10 Tage mit 13 Laboratorien im Linzer-Raum durchgeführt und die Prazision und Richtigkeit an Hand des Kontrollblutes CH-35 (DADE) für Counter- und Kammerzählungen untersucht (1). Geprüft wurden die Erythrozyten, die Leukozyten und das Hämoglobin. Wir fanden für die Erythrozytenzählung im Counter $(N=232)$ im Normbereich einen VK von $3,6 \%$, für die Kammer $8,8 \%$, für die Leukozytenzählung für den Counter $9 \%$, für die Kammer $12 \%$, das Hämoglobin war mit $3 \%$ sehr zufriedenstellend. Im abnormalen Bereich wurden ebenfalls sehr gute Ergebnisse erzielt. Nähere Einzelheiten dieses Versuchs mögen aus der Publikation entnommen werden.

Auch für die Thrombozytenmessung wurden regionale Rundversuche durchgeführt. Getestet wurde das plättchenangereicherte Plasma HAEM-PC im normalen und abnormalen Bereich (2). Hier war die Präzision im Vergleich zu dem eben angeführten Rundversuch für die Countermessung im Normbereich gleichwertig, im abnormalen Bereich hingegen betrug der VK $13 \%(\bar{x}=78,8$ G/I). Bei den Kammerzählungen betrugen die VKs zwischen $9 \%$ und $14 \%$.

Der regionale Rundversuch, ganz allgemein gesprochen, ist dem nationalen vorzuziehen. Es kommt ihm deswegen eine größere Bedeutung zu, da durch persönliche Kontakte und Diskussionen eine aktive Mitarbeit erreicht wird und damit die Ursachen fehlerhafter Ergebnisse untersucht und die Fehler behoben werden können.

\section{Nationale Rundversuche}

Die externen nationalen Rundversuche werden in Österreich seit 1980 institutionalisiert, von der ÖQUASTA durchgeführt und sowohl Klinische Chemie als auch Gerinnung und Hämatologie werden erfaßt.

In der Tab. 3 sind die Resultate der VKs über den gesamten Zeitraum zwischen 1980 und 1982 (insgesamt 7 Rundversuche) für die Erythrozyten, Leukozytenmessung, für das Hämoglobin und den Hämatokrit als Verlaufskontrolle übersichtlich zusammengefaßt.

Untersucht werden im nationalen Rundversuch immer zwei Proben mit unterschiedlichem Meßbereich, wobei sich hier naturgemäß Einflüsse von seiten des Mittelwertes bemerkbar machen.

Die Präzision der Erythrozytenmessung liegt für die Leitfähigkeitsmessung besser im Vergleich zur Kammerzählung, das optische Verfahren (Laserstrahltechnik) kommt der Leitfähigkeitsmessung nahe und die Fotometrie (Gowersche Lösung) der Kammerzählung. Für die Leukozyten erreicht das optische Verfahren (wenig Rundversuchsteilnehmer) einen guten VK, die Zählkammer ist naturgemäß schlechter im Vergleich zur Leitfähigkeitsmessung. Für die Hämoglobinbestimmung wird fast ausschließlich die Hämoglobinzyanidmethode angewandt und man muß mit einem globalen Ergebnis von $5 \%$ durchaus zufrieden sein.

Für die Hämatokritmessung liegt die Referenzmethode (= Zentrifugen) im Vergleich zu den Methoden der "Berechnung" "und "Leitfähigkeitsmessung" wesentlich besser.

Auch die Frage nach einer Diagnose aus dem Differentialblutbild wird gestellt. Wir haben zur Demonstration zwei Rund-
Tab. 3: Ergebnisse (VK\%) der OQUASTA-Rundversuche für Hämoglobin. Erythrozyten, Leukozyten und Hämatokrit

\begin{tabular}{lrr}
\hline Methode & $N$ & $V K(\%)$ \\
\hline 1. Hämoglobincyanid & 14 & 5,11 \\
2. Erythrozytenzählung: & & \\
a) Leitfähigkeit & 14 & 4,6 \\
b) Zählkammer & 14 & 9,7 \\
c) Optisches Verfahren & 14 & 5,1 \\
d) Photometer & 8 & 8,5 \\
& & \\
3. Leukozytenzählung: & 14 & 11,5 \\
a) Leitfähigkeit & 14 & 34,0 \\
b) Zählkammer & 12 & 6,9 \\
c) optisches Verfahren & & \\
Hämatokrit & 12 & 5,6 \\
a) Leitfähigkeit & 14 & 9,5 \\
b) Zentrifuge & 14 & 7,2 \\
c) Berechnung & & \\
\hline
\end{tabular}

versuche, einen mit einem Normalbefund und einen mit der Diagnose "Chron. Lymph. Leukämie" ausgewählt. Dabei zeigt sich beim Normalbefund eine wesentlich größere Unsicherheit in der Diagnosestellung als im pathologischen Fall z. B. der chron. lymph. Leukämie.

Bei der Analyse der Resultate aus den Protokollbögen ergaben sich Schwierigkeiten für die Interpretation, da manche Laboratorien über das Prinzip der angewandten Methode nicht vollkommen informiert sind.

Anerkennung:

Für die Überlassung der Resultate mit dem Kontrollblut „Control Blood Plus" der Firma DADE möchten wir Frl. Franziska Tenger (Fa. Dade, $\mathrm{CH}-4186$ Düdingen) sehr herzlich danken.

Schrifttum:

1. HOHENWALLNER, W., WIMMER, E.: Qualitätskontrolle hämatologischer Parameter mit Hilfe eines stabilisierten Kontrollblutes. Ärztl. Lab. 21, 42-48 (1975).

Hilfe eines stabilisierten Kontrollblutes. Arztl. Lab. 21, $42-48$ (1975). 2. HOHENWALLNER, W., WIMMER, E., SOMMER, R.: Qualitätsk

tenzählung mit HAEM-PC. Berichte der OGKC 3, 34-36 (1980). 3. WIDER, G., KOHN, H., BAYER, P. M.: Hămatologische Referenzbereiche
für den Blutersatz. Das Medizinische Laboratorium 35, 86-89 (1982).
Anschrift des Verfassers:

Prim. Dr. W. Hohenwallner

Zentrallaboratorium

$\mathrm{KH}$ der Barmherzigen Schwestern

Langgasse 16

A-4020 Linz 